\title{
Electrohydraulic lithotripsy of large bile duct stones under direct cholangioscopy with a double-balloon endoscope
}

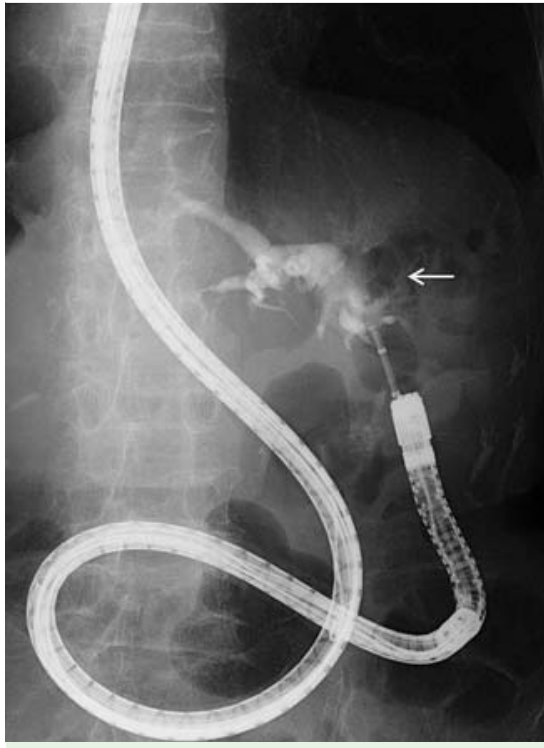

Fig. 1 Cholangiography with a short doubleballoon endoscope delineates large bile duct stones (arrow) in the right hepatic duct of an 82-year-old woman with a history of hepaticojejunostomy.

The use of a short double-balloon endoscope facilitates the endoscopic removal of bile duct stones in patients with surgically altered anatomy $[1,2]$. However, the narrow and long working channel of the double-balloon endoscope occasionally makes the procedure difficult and timeconsuming. Herein, we present the case of a patient with a history of hepaticojejunostomy in whom large bile duct stones were successfully fragmented and removed under direct visualization with a short double-balloon endoscope and electrohydraulic lithotripsy (EHL).

An 82-year-old woman with a history of hepaticojejunostomy and Roux-en-Y reconstruction for congenital biliary dilatation was admitted with cholangitis due to intrahepatic bile duct stones. Endoscopic retrograde cholangiopancreatography (ERCP) was carried out with a short double-balloon endoscope (EC-450BI5, 2.8-mm-wide and 152-cm-long working channel; Fujifilm Corp., Tokyo, Japan), and biliary access was readily obtained with a usual ERCP cannula and a 0.025-in guidewire. Cholangiography delineated
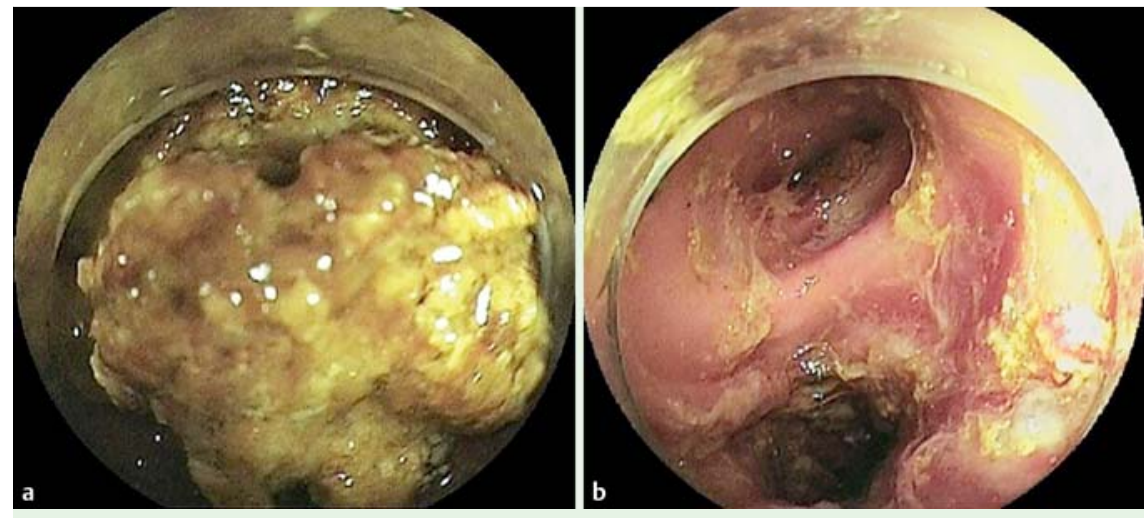

Fig. 2 a Direct cholangioscopy with a double-balloon endoscope reveals large bile duct stones in the right hepatic duct. b Successful fragmentation of the bile duct stones with electrohydraulic lithotripsy.

an 18-mm filling defect in the right hepatic duct $(\bullet$ Fig. 1, $\bullet$ Fig. 2 a).

After attempts to extract the bile duct stones with a retrieval balloon and basket catheters had failed, EHL was performed with the AUTOLITH System and a 1.9-Fr, 250-cm-long probe (Northgate Technologies, Elgin, Illinois, USA). The distal end of the double-balloon endoscope was inserted into the bile duct, and water irrigation for EHL was achieved by pushing the balloon against the anastomosis and flushing saline through the channel. Large stones were successfully fragmented with EHL, followed by complete removal with a balloon catheter ( Fig.2b, Video 1 ). No procedure-related complications were observed.

This is the first report of successful stone fragmentation with EHL under direct visualization with a short double-balloon enteroscope. The effectiveness of $\mathrm{EHL}$ combined with a single-balloon endoscope has been reported [3], and given the potential superiority of a double-balloon enteroscope over a single-balloon enteroscope in terms of endoscope insertion [4], EHL with a double-balloon enteroscope can further increase the technical success rate of stone removal in patients with surgically altered anatomy.

\section{Endoscopy_UCTN_Code_TTT_1AR_2AH}

\section{Competing interests: None}

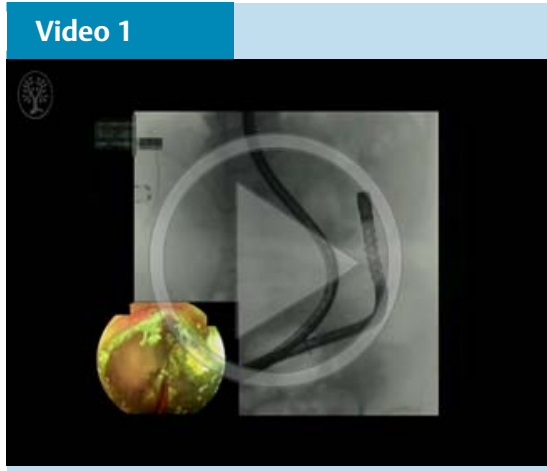

Electrohydraulic lithotripsy combined with double-balloon endoscopy for large bile duct stones in a patient with a history of hepaticojejunostomy.

Ryunosuke Hakuta, Hirofumi Kogure, Hiroyuki Isayama, Atsuo Yamada, Tsuyoshi Hamada, Yousuke Nakai, Kazuhiko Koike

Department of Gastroenterology, Graduate School of Medicine, University of Tokyo, Tokyo, Japan

\section{References}

1 Aabakken L, Bretthauer M, Line PD. Doubleballoon enteroscopy for endoscopic retrograde cholangiography in patients with a Roux-en-Y anastomosis. Endoscopy 2007; 39: $1068-1071$

2 Tsujino T, Yamada A, Isayama $\mathrm{H}$ et al. Experiences of biliary interventions using short double-balloon enteroscopy in patients with Roux-en-Y anastomosis or hepaticojejunostomy. Dig Endosc 2010; 22: 211-216 
3 Kao KT, Batra B. Single-balloon-assisted ERCP with electrohydraulic lithotripsy for the treatment of a bile duct stone in a patient with a hepaticojejunostomy. Gastrointest Endosc 2014; 80: 1173

4 Takano N, Yamada A, Watabe $H$ et al. Singleballoon versus double-balloon endoscopy for achieving total enteroscopy: a randomized controlled trial. Gastrointest Endosc 2011; 73: 734-739

\section{Bibliography}

Dol http://dx.doi.org/

10.1055/s-0034-1392669

Endoscopy 2015; 47: E519-E520

(c) Georg Thieme Verlag KG

Stuttgart · New York

ISSN 0013-726X

\section{Corresponding author} Hiroyuki Isayama, MD, PhD

Department of Gastroenterology Graduate School of Medicine University of Tokyo

7-3-1 Hongo Bunkyo-ku Tokyo

Japan 113-8655

Fax: +81-3-3814-0021

isayama-tky@umin.ac.jp 